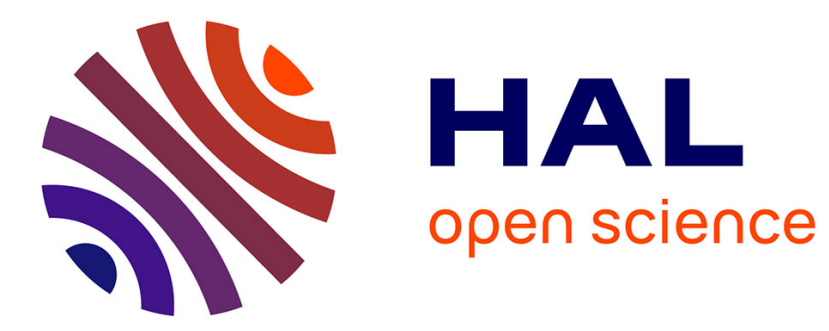

\title{
L'approche moderne du pied de Charcot
}

\author{
A. Hartemann
}

\section{To cite this version:}

A. Hartemann. L'approche moderne du pied de Charcot. Médecine des Maladies Métaboliques, 2016, 10 (6), pp.515 - 518. 10.1016/S1957-2557(16)30164-X . hal-01401609

\section{HAL Id: hal-01401609 https://hal.sorbonne-universite.fr/hal-01401609}

Submitted on 23 Nov 2016

HAL is a multi-disciplinary open access archive for the deposit and dissemination of scientific research documents, whether they are published or not. The documents may come from teaching and research institutions in France or abroad, or from public or private research centers.
L'archive ouverte pluridisciplinaire HAL, est destinée au dépôt et à la diffusion de documents scientifiques de niveau recherche, publiés ou non, émanant des établissements d'enseignement et de recherche français ou étrangers, des laboratoires publics ou privés. 


\section{Dossier thématique "Le pied diabétique »}

\section{L'approche moderne du pied de Charcot}

The modern approach of Charcot foot

\section{A. Hartemann}

Service de diabétologie, Groupe hospitalier Pitié-Salpêtrière, AP-HP, Paris ; Université Paris6 ; Institut E3M, IHU ICAN, Paris.

\section{Agnès Hartemann}

Service de diabétologie

Groupe hospitalier Pitié-Salpêtrière

83 boulevard de l'Hôpital

75651 Paris cedex 13.

agnes.hartemann@aphp.fr 


\section{Résumé}

On comprend mieux actuellement la physiopathologie du «pied de charcot». Sur un pied ou une cheville neuropathique survient un microtraumatisme responsable d'une inflammation locale qui, si elle perdure, va entraîner une activation des ostéoclastes avec lyse ostéo-articulaire. Une IRM permet de faire le diagnostic précoce, et de mettre rapidement le pied en décharge afin de stopper ce processus. Les biphosphonates ne sont plus recommandés. Faire le diagnostic à la phase aiguë précoce (appelée aussi phase active) permet de préserver la statique du pied. C'est la sémiologie, le diagnostic et le traitement de cette phase aiguë qui devrait être enseignés et non plus l'historique pied cubique fixé qui n'a aucun intérêt pour le soin du patient.

Mots-clés :pied de charcot; neuropathie; diabète

SummaryPhysiopathology of « charcot foot» is now more and more clear. In presence of neuropathy, a little trauma at the level of foot or ankle induces inflammation. The latter, if lasting, is responsible for activation of the osteoclast system which leads to the lysis of bones and joints. MRI can make an early diagnosis. Then quick off-loading can prevent the destruction of bones and joints. Biphosphonates are no more recommended. It is important to make diagnosis at the active early phase to keep the foot and ankle intact. This is the semiology, diagnosis and treatment of this active early phase that should be teach, and no longer the historical "rocker bottom foot" which have no interest for the patient well-being.

Key-words:charcot foot ; neuropathy ; diabetes 
Les points essentiels

Le pied de Charcot est une neuro-osteoarthropathie qui peut toucher aussi la cheville. Un micro-traumatisme sur un pied ataxique et insensible entraîne une inflammation qui peut conduire à la lyse osseuse, sauf si l'IRM précoce fait le diagnostic, et qu'une suppression de l'appui est mise en place. Plusieurs phases actives peuvent se succéder. Il faut savoir les reconnaître et les traiter pour éviter la destruction ostéo-articulaire. 


\section{Introduction}

La dislocation ostéo-articulaire du pied de Charcot a été décrite, en 1883, par Jean-Martin Charcot, et c'est un anglais, Herbert William Page, qui fit, la même année, le lien avec la neuropathie périphérique [1]. Récemment, la connaissance de la physiopathologie a progressé. Un diagnostic précoce est possible avec une imagerie par résonance magnétique (IRM). Il est donc temps de cesser d'enseigner le pied de Charcot comme un «gros pied cubique », mais parler de la phase aiguë, et du traitement qui évite la dislocation. D'autant que cette phase aiguë peut se répéter, avec le même risque à chaque fois. On parle plus volontiers dorénavant d'ostéoarthropathie neuropathique en phase active ou inactive.

\section{Une nouvelle définition}

* Le «pied de Charcot» est un processus complexe multifactoriel qui ne survient qu'en présence d'une neuropathie, et peut aussi atteindre la cheville, et même parfois le genou. Il est donc recommandé de parler maintenant plutôt d'ostéoarthropathie neuropathique de Charcot [2]. Le processus implique les os, les articulations et les tissus mous et, typiquement, une phase inflammatoire précoce.

* On parlait antérieurement d'une phase aigüe inflammatoire pendant laquelle survenait une destruction de certains segments osseux et des subluxations articulaires, conduisant à une dislocation. Puis, d'une phase chronique où le pied se stabilisait dans un état déformé, mais «calé » par une reconstruction osseuse anarchique. Comme si l'histoire était linéaire et unique. On sait maintenant que cette succession «phase inflammatoire-destruction-fixation des déformations » peut se répéter sur différents segments ostéo-articulaires. On peut voir, par exemple, un patient avec un pied cubique ancien développer soudainement une inflammation, 
puis une ostéoarthropathie de la cheville. On préfère donc utiliser les termes de «phase active » et «phase inactive », en sachant que les phases actives peuvent se répéter.

\section{Une nouvelle compréhension physiopathologique}

* Dans la mesure où l'ostéoarthropathie de Charcot survient toujours sur un pied neuropathique, parfois hyper-vascularisé par des shunts artério-veineux, on a longtemps pensé que l'augmentation du flux sanguin était à l'origine d'une déminéralisation expliquant l'apparition soudaine de la destruction ostéo-articulaire. Mais, ceci n'expliquait pas pourquoi l'atteinte pouvait rester très longtemps unilatérale, et la déminéralisation ne semble associée qu'avec les manifestations fracturaires pures et pas les dislocations [3]. De plus, on retrouve plus souvent une déminéralisation chez les patients diabétiques de type 1 que de type 2, alors qu'ils ne sont pas plus concernés par l'ostéoarthropathie de Charcot.

On disait aussi qu'on ne pouvait pas voir, de ce fait, une ostéoarthropathie en présence d'une artériopathie des membres inférieurs, puisque le flux sanguin dans ce contexte est réduit, ce qui s'avère inexact: on peut voir le processus se développer sur un «pied mixte » neuropathique et artériopathique.

* Il semble, en fait, que le développement de la neuro-ostéoarthropathie soit multifactoriel. La neuropathie sensitive est le terrain de base, quelle que soit son origine (diabète, alcool, chimiothérapie, lèpre...). Sur ce terrain favorisant l'instabilité à la marche (ataxie proprioceptive) et les troubles morphostatiques, surviendrait un traumatisme mineur à la marche, dont se souviennent de nombreux patients lorsqu'on les interroge [4]. Certaines équipes suggèrent même qu'une plaie ou qu'une ostéite pourrait faire partie de ces traumatismes initiaux, mais ceci est très discuté. Il est tout à fait possible que ce microtraumatisme survienne sur un pied préalablement déminéralisé, expliquant la survenue 
parfois de phases actives de neuro-arthropathie à la reprise d'un appui après une phase d'immobilisation (celle-ci favorisant la déminéralisation).

- Ce traumatisme entrainerait une réaction locale inflammatoire avec relargage local de tumor necrosis factor (TNF)- $\alpha$ et d'interleukine (IL)-1ß, conduisant à l'augmentation de l'expression de RANK ligand (RanKl, pour receptor activator of nuclear factor kappa-B ligand). RANKl favorise la synthèse du facteur nucléaire nuclear factor-kappa $B(\mathrm{NF}-\kappa \mathrm{B})$, qui stimule la maturation des ostéoclastes et l'ostéolyse. Le patient neuropathique continuerait, dans ce contexte, à déambuler, puisque l'inflammation ne le fait pas ou peu souffrir, alors que, sans neuropathie, il s'immobiliserait. Il favorise ainsi la répétition ou l'aggravation des microtraumatismes, entretenant la réaction inflammatoire et l'activation des ostéoclastes [5].

Une étude très élégante a montré la capacité du sérum de patients présentant une neuroarthropathie à la phase active, à activer des ostéoclastes in vitro [6]. TNF- $\alpha$ semble capable d'être à l'origine de l'activation des ostéoclastes par RANKl [7].

Le phénomène finirait pas s'auto-réguler, car NFkB stimule la production d'ostéoprotégérine qui est un antagoniste de RANKl. Certains peptides normalement synthétisés par les extrémités nerveuses, comme le «calcitonin gene-related peptide »(CGRP), sont aussi des antagonistes de RANK1. Ils pourraient faire défaut en présence d'une neuropathie, laissant le système s'activer de manière prolongée sans s'autoréguler lors d'une inflammation.

\section{Le diagnostic moderne du pied de Charcot}

* La neuro-ostéoarthropathie devrait être diagnostiquée au stade le plus précoce. Le patient consulte car, malgré la neuropathie, il a ressenti une douleur au niveau de son pied, et/ou a 
remarqué que celui-ci était gonflé et chaud. Il peut raconter avoir ressenti une douleur à la marche, ou s'être «tordu la cheville ». À l'examen, son pied est en effet œeématié, rosé et chaud (figure 1). La neuropathie sensitive est évidente : le patient ne sent pas le toucher, ni le monofilament. Dans la grande majorité des cas, il n'a pas de plaie. Cet aspect peut concerner aussi la cheville, et ressembler alors à une légère entorse.

* Le médecin qui a la chance de voir le patient à ce stade peut sauver son pied ou sa cheville de la dislocation. Tout pied (ou cheville) neuropathique chaud gonflé et rosé est, en effet, un «pied de Charcot » jusqu'à preuve du contraire. L'examen à demander immédiatement est une radiographie, voire, si possible, d'emblée une IRM. La radiographie peut être encore normale, ou montrer déjà une fracture. Dans $70 \%$ des cas, cette fracture se situe au niveau des articulations tarso-métatarsales (typiquement entre la $1^{\text {re }}$ et la seconde : figure 2), ou au niveau des os du tarse. Plus rarement, au niveau de l'avant-pied (métatarses, articulations métatarso-phalangiennes), de la cheville, ou du calcanéum.

- Si la radiographie est normale, ou si possible d'emblée, l'IRM va montrer beaucoup plus précocement un œdème intra-osseux (figure 3) et une (ou des) microfractures (figure 4). Il vaut mieux préciser au radiologue les signes que l'on recherche (œè̀me médullaire et fractures), car «suspicion de pied de Charcot» ne leur évoque souvent rien. Au mieux, le diagnostic d'algodystrophie est évoqué, car les aspects sont comparables. Cet aspect à l'IRM suffit à affirmer le diagnostic.

- La radiographie ou l'IRM, associées à la clinique, suffisent donc à poser le diagnostic. Il n'y a pas actuellement de marqueurs biologiques. À ce stade, le traitement va éviter la dislocation.

- Si l'IRM est strictement normale, on peut éliminer le diagnostic. 
- Lorsque, malheureusement, le diagnostic est fait après la survenue de la dislocation de l'arche plantaire ou de la cheville, le diagnostic est évident à la radiographie. Si le pied est encore en phase active (inflammatoire), le traitement évitera simplement l'aggravation.

\section{Le traitement de la neuro-ostéoarthropathie}

* Suite à une étude ouverte, on a cru un temps que les biphosphonates pouvaient être efficaces. Mais, deux études randomisées ont prouvé que ce n'était pas le cas [8,9], voire qu'ils pourraient être délétères. Ils sont donc contre-indiqués [10]. La découverte de l'implication du système TNF-RANKl pourrait ouvrir des perspectives thérapeutiques.

* Le seul traitement efficace reste, à l'heure actuelle, la mise en décharge et la protection du pied par le système utilisé. Le but est de rompre le cercle vicieux de l'inflammation-activation des ostéoclastes, et de protéger les os tant qu'ils sont vulnérables. Les anglo-saxons utilisent un plâtre de décharge inamovible, mais la botte ou la bottine «air-cast», si elle est portée pour le moindre pas, est efficace. Le patient peut marcher un peu avec (ce qui évite la déminéralisation), mais doit être en arrêt de travail.

- Lorsque le patient voit sur Internet les conséquences de la dislocation du «pied de Charcot », et qu'il comprend qu'il a été pris à temps au stade de simple fracture, il est en règle, au début, assez motivé pour porter la bottine. La durée de la mise en décharge est de 4 à 6 mois. Le critère d'interruption est la disparition des signes d'inflammation locale (œdème, chaleur) à la remise en charge (figure 1). On peut s'aider d'un thermomètre, qui permet de vérifier qu'il n'y a plus de différence de température cutanée entre les deux pieds (à la phase active, cette différence est d'au moins $2^{\circ} \mathrm{C}$ ). Aucune étude n'a évalué l'intérêt de l'IRM dans le suivi. 


\section{L'enseignement de cette complication}

* Les médecins généralistes sont en première ligne pour faire le diagnostic de neuroostéoarthropathie à la phase active. Cette pathologie est rare, mais, surtout, elle est mal enseignée. On présente toujours le classique «pied cubique fixé » en photographie marquante aux étudiants. De ce fait, les médecins généralistes, devant un pied ou une cheville inflammatoire, évoquent, à juste titre, une phlébite, une crise de goutte, ou une infection. Le patient prend de la colchicine, fait un écho-doppler veineux, bande sa cheville, et continue de marcher, jusqu'à la survenue en 2 à 3 semaines (mais parfois plus tard) de la tragique dislocation ostéo-articulaire irréversible.

* Nous devons donc changer nos messages et enseigner que « tout pied gonflé chez un patient diabétique doit faire recherche une neuropathie », et qu'en présence de celle-ci, il s'agit d'un «pied de Charcot en phase active jusqu'à preuve du contraire ». L'immobilisation dans une botte air-cast doit être immédiate. Rien n'empêche, par ailleurs, le patient de faire un échodoppler veineux, mais une IRM du pied en urgence, à la recherche d'un œedème médullaire et de fractures, doit faire partie du bilan.

* Enfin, dans les programmes de prévention à l'intention des patients de grade 1 à 3 , le message doit aussi être passé : le patient doit consulter en urgence, et se mettre au repos devant un pied qui gonfle, même sans plaie.

\section{Déclaration d'intérêt}

L'auteure déclare 


\section{Références}

1. Sanders LJ, Edmonds ME, Jeffcoate WJ. Who was first to diagnose and report neuropathic arthropathy of the foot and ankle: Jean-Martin Charcot or Herbert William Page? Diabetologia 2013;56:1873-7.

2. Rogers LC, Frykberg RG, Armstrong DG, et al. The Charcot foot in diabetes. Diabetes Care 2011;34:2123-9.

3. Herbst SA, Jones KB, Saltzman CL. Pattern of diabetic neuropathic arthropathy associated with the peripheral bone mineral density. J Bone Joint Surg $\mathrm{Br}$ 2004;86:378-83.

4. Foltz KD, Fallat LM, Schwartz S. Usefulness of a brief assessment battery for early detection of Charcot foot deformity in patients with diabetes. J Foot Ankle Surg 2004;43:87-92.

5. Jeffcoate WJ, Game F, Cavanagh PR. The role of proinflammatory cytokines in the cause of neuropathic osteoarthropathy (acute Charcot foot) in diabetes. Lancet 2005;366:2058-61.

6. Mabilleau G, Petrova NL, Edmonds ME, Sabokbar A. Increased osteoclastic activity in acute Charcot's osteoarthropathy: the role of receptor activator of nuclear factorkappaB ligand. Diabetologia 2008;51:1035-40.

7. Petrova NL, Petrov PK, Edmonds ME, Shanahan CM. Inhibition of TNF- $\alpha$ reverses the pathological resorption pit profile of osteoclasts from patients with acute Charcot osteoarthropathy. J Diabetes Res 2015;2015:917945.

8. Jude EB, Selby PL, Burgess J, et al. Bisphosphonates in the treatment of Charcot neuroarthropathy: a double-blind randomised controlled trial. Diabetologia 2001;44:2032-7. 
9. Pakarinen TK, Laine HJ, Mäenpää $H$, et al. The effect of zoledronic acid on the clinical resolution of Charcot neuroarthropathy: a pilot randomized controlled trial. Diabetes Care 2011;34:1514-6.

10. Richard JL, Almasri M, Schuldiner S. Treatment of acute Charcot foot with bisphosphonates: a systematic review of the literature. Diabetologia 2012;55:1258-64.

Figures 


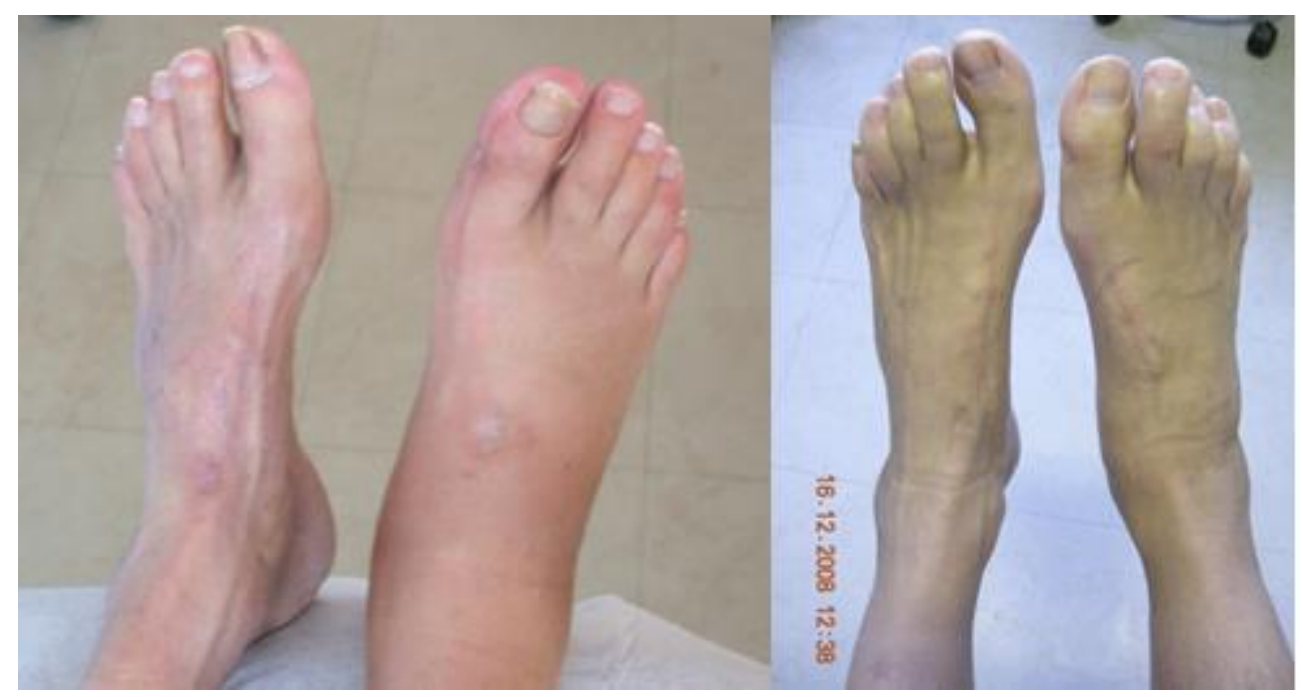

\section{Figure 1.}

- À gauche : phase active de neuro-ostéoarthropathie de Charcot au niveau du pied droit.

- À droite : disparition des signes d'inflammation après 6 mois de mise en décharge. 


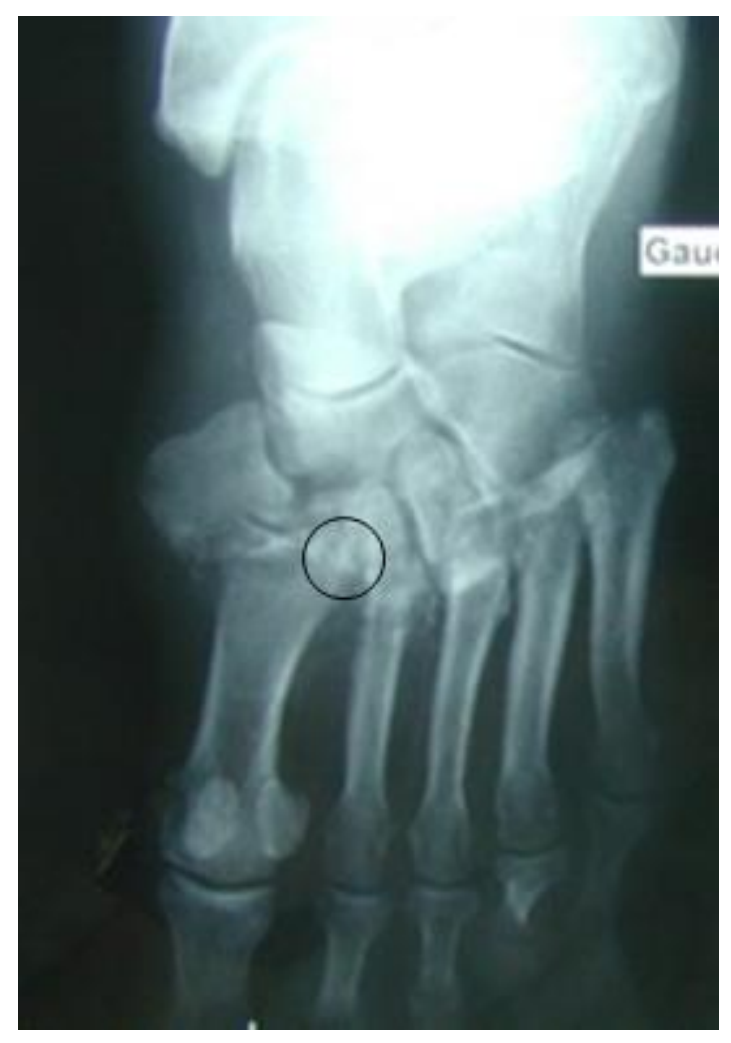

Figure 2. Fracture à la base du $1^{\mathrm{er}}$ métatarsien à la phase active.

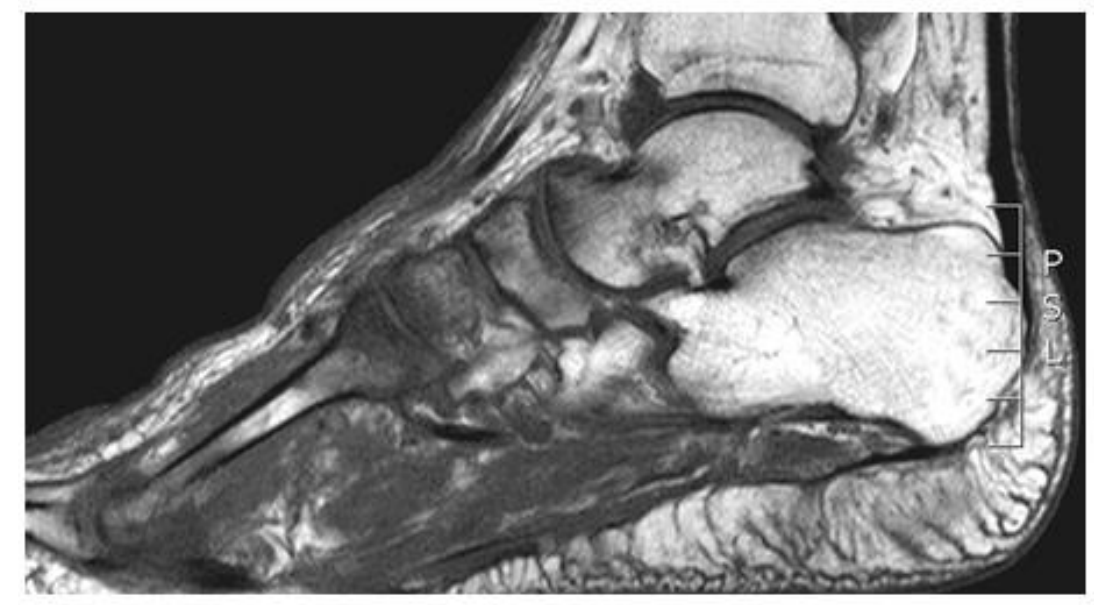

Figure 3. Edème médullaire du tarse à la phase active. 

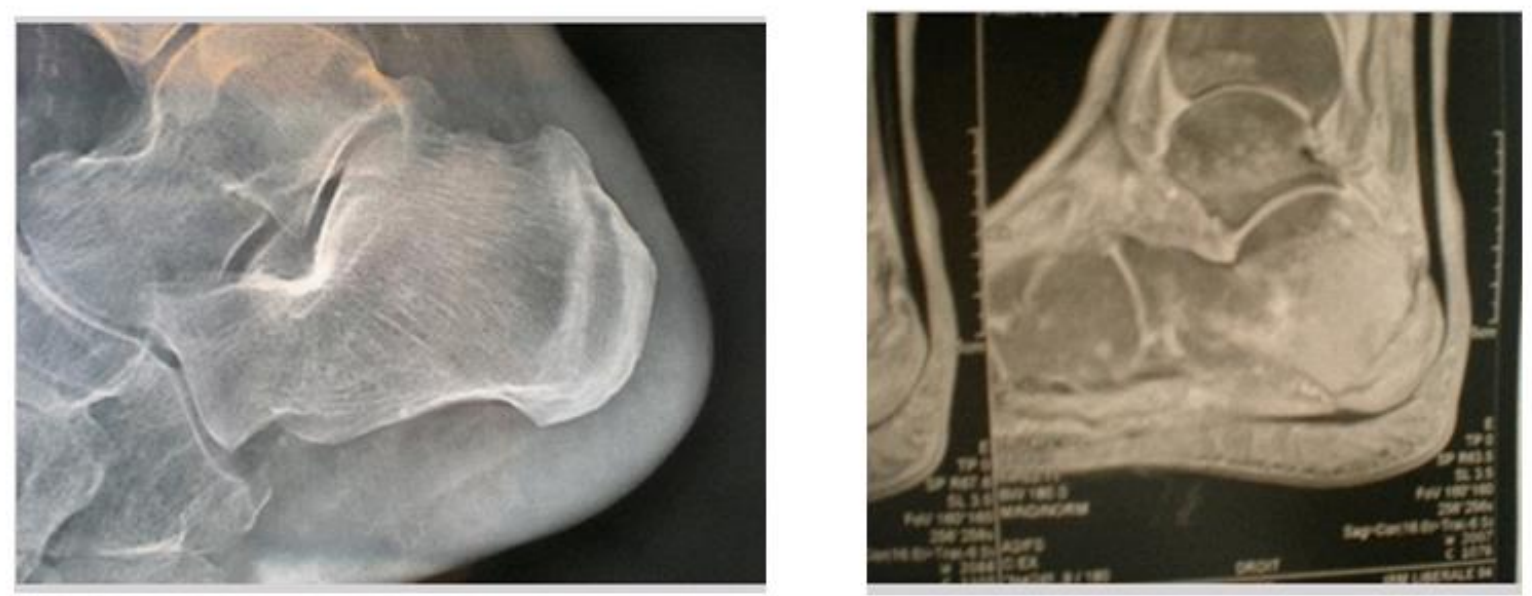

Figure 4. Fracture du calcanéum à la phase active : visible sur l'imagerie par résonance magnétique (IRM), à droite ; non visible sur la radiographie, à gauche. 